\title{
5. Diskussion und Ausblick
}

Die Daten der KFN-Schülerbefragung 2005 zur Mediennutzung von Kindern und Jugendlichen bestätigen und ergänzen Befunde anderer aktueller Studien, die in Kapitel 2 vorgestellt wurden. Jungen der vierten wie auch der neunten Klassen sind deutlich besser mit eigenen Mediengeräten ausgestattet als Mädchen und nutzen elektronische Medien insgesamt auch intensiver. Dies gilt im Grundschulalter sowohl für Spielkonsolen, Computer als auch für den Fernseher. Wenn auch in der neunten Klasse die Ausstattungsunterschiede bei all diesen Geräten zwischen den Geschlechtern weiterhin bestehen, stellen sich die Geschlechterdifferenzen bei den Nutzungszeiten etwas anders dar. Während die Fernsehzeiten von Jungen und Mädchen fast identisch sind, spielen Jungen immer noch deutlich häufiger und länger Computer- bzw. Konsolenspiele. Darüber hinaus verbringen sie auch mehr Zeit im Internet.

Insgesamt, auch hier zeigen sich Parallelen zu anderen Studien, nimmt Medienzeit mit höherem Alter der Kinder bzw. Jugendlichen einen immer breiteren Raum im Alltag von Schülern ein. Dies gilt geschlechterübergreifend. Besonders das Internet, dessen Bedeutung erst nach dem Grundschulalter steigt, trägt signifikant zu einer Steigerung der Gesamtmedienzeit bei.

Im Einklang mit anderen Studien zeigen auch die KFN-Daten, dass Jungen eine deutlich höhere Affinität zu Mediengewaltinhalten besitzen als Mädchen. Dies gilt über alle Mediengrenzen hinweg. Bei Filmen im Fernsehen wie auch bei Computerspielen ist der Anteil von Mädchen, die gelegentlich oder häufig Mediengewaltinhalte nutzt marginal. Im Medienmenü der Jungen spielen Gewaltinhalte sowohl in der vierten als auch in der neunten Klasse eine wichtige Rolle.

Ebenfalls analog zu anderen Studien zeigt die KFN-Schülerbefragung, dass eine gute Ausstattung mit Mediengeräten und eine intensive Nutzung von Bildschirmmedien einen recht zuverlässigen Indikator für den sozioökonomischen Hintergrund einer Familie und das Bildungsniveau im Elternhaus von Schülerinnen und Schülern darstellen. „Medienfreizeit“" besitzt vor allem in solchen Familien einen hohen Stellenwert, für die ein formal niedriger Bildungsstand der Eltern und nicht selten Arbeitslosigkeit oder Sozialhilfebezug kennzeichnend sind. Interessant ist an dieser Stelle, dass sich je nach Bildungsniveau im Elternhaus bereits bei Vierklässlern deutliche Unterschiede in der Selektion von Medieninhalten zeigen. Kinder aus formal niedrig gebildeten Familien neigen beispielsweise eher zu Angeboten privater Fernsehstationen, während Viertklässler aus formal hoch gebildeten Elternhäusern viel häufiger öffentlich-rechtliche Angebote wie den Kinderkanal als ihren Lieblingssender angeben.

Zusätzlich zu den oben berichteten Befunden weisen die Daten der KFNSchülerbefragung auf einige weitere Punkte hin, die bisher in der Debatte über die Mediennutzung von Kindern und Jugendlichen kaum Beachtung gefunden haben. So 
zeigt sich im Vergleich der Mediennutzungsdaten verschiedener Regionen neben dem bereits berichteten Geschlechtereffekt auch ein deutliches Nord-Süd-Gefälle in der Mediennutzung. Kinder und Jugendliche aus süddeutschen Befragungsgebieten wiesen relativ niedrige Mediennutzungszeiten und eine deutliche geringere Mediengeräteausstattung auf. Obwohl diese Befunde durch die Fokussierung der Studie auf einige wenige Befragungsgebiete nicht den Anspruch haben können, ein deutschlandweites Phänomen zu beschreiben, sollten sie aufgrund der teilweise großen interregionalen Unterschiede zumindest Anlass geben, nach regionalen Besonderheiten zu fragen, die diese Unterschiede erklären können.

Neben dem höheren formalen Bildungsniveau im Elternhaus, das in den eher städtisch geprägten süddeutschen Befragungsgebieten mit Sicherheit einen Teil der regionalen Mediennutzungsunterschiede erklären kann, sollten bei der Interpretation der Befunde auch sozialstrukturelle Merkmale berücksichtigt werden, die durch die KFN-Schülerbefragung nicht vollständig erfasst werden konnten. So liegt die Vermutung nahe, dass niedrige Mediennutzungszeiten vor allem in solchen Gebieten die Regel sind, in denen traditionelle soziale Strukturen wie ein breites strukturiertes Freizeitangebot in Sportvereinen oder Orchestern die Regel sind und in denen es gelingt, möglichst alle Schichten der Bevölkerung in solche Strukturen zu integrieren. Medienfreizeit scheint häufig - trotz aller auf anspruchsvollen Kinder- und Jugendmedien beruhenden Hoffnungen - nur eine Kompensation fehlender alternativer Angebote zu sein. In Gebieten, in denen Kindern und Jugendlichen solche Alternativen mutmaßlich zur Verfügung stehen, verliert Mediennutzung deutlich an Bedeutung.

Ein weiterer wichtiger Aspekt, der durch die KFN-Schülerbefragung erstmals derart differenziert erforscht wurde, ist die Nutzung entwicklungsbeeinträchtigender Medien durch Kinder und Jugendliche. Besonders für den Bereich der Computerspiele konnte durch die offene Abfrage derzeit genutzter Spieltitel gezeigt werden, dass Computerspiele mit einer Alterkennzeichnung ,ab 16“ oder „keine Jugendfreigabe" bereits im Medienalltag männlicher Viertklässler eine wichtige Rolle spielen. Darüber hinaus kann verdeutlicht werden, dass der Besitz eigener Mediengeräte im Zimmer die Nutzung solcher Inhalte sehr begünstigt. Besonders bei Jungen, die mit einem älteren Bruder ein Zimmer teilen, zeigt sich dieser Zusammenhang. Elterliche Medienerziehung kann an dieser Stelle ein wichtiger Protektor vor der Nutzung solcher Inhalte sein und darüber hinaus eine zeitlich ausufernde Nutzung von Bildschirmmedien in vernünftigen Grenzen halten. Dass elterliche Medienerziehung dagegen häufig nicht stattfindet - auch und gerade dort nicht, wo Eltern aufgrund von Arbeitslosigkeit die Zeit dazu hätten - hängt mutmaßlich auch mit einem anderen Aspekt zusammen: Qualität und Quantität von Mediennutzung scheinen insgesamt Kennzeichnen bestimmter Freizeit- und Bildungsmilieus zu sein, was auch in der Forschung zum Freizeitverhalten von Kindern und Jugendlichen stärker berücksichtigt werden muss. Fehlende Medienerziehung durch die Eltern und problematische Mediennutzung der Kinder korrelieren nicht nur, sondern sind Kennzeichen eines bestimmten Lebensstils.

Vor diesem Hintergrund eine kompetente Mediennutzung vieler Kinder und Ju- 
gendlicher zu erwarten oder auf förderliche Mediennutzungseffekte durch eine stärkere Hinwendung zu interaktiven Lernmedien oder dem breiten Informationsangebot des Internets zu setzen, scheint eher naiv zu sein. Genauso wie Medienkompetenz durch elterliche Vorbilder vermittelt wird, scheint sich auch unreflektierte, problematische Mediennutzung über elterliche (Nicht-)Vorbilder zu vermitteln und zu verfestigen. Dies ist auch dann der Fall, wenn neue Medienformate wie Computerspiele Einzug in die Kinder- und Jugendzimmer halten. Auch in diesem Bereich, in dem Eltern aller Bildungsniveaus und Einkommensklassen wenig oder gar keine eigene Erfahrung vorweisen können, zeigen sich doch deutliche Unterschiede elterlicher Medienerziehung je nach dem sozialen Milieu, in dem die Kinder groß werden.

Betrachtet man den Zusammenhang von Mediennutzung und Schulleistung ergeben die dargestellten internationalen Studien zusammengefasst das Bild eines negativen Einflusses von Ausstattung und Inhalten auf die Schulleistungen von Kindern und Jugendlichen (vgl. Kapitel 2). Hinsichtlich der Erklärung zugrunde liegender Wirkmechanismen herrscht jedoch in der Forschung noch eine gewisse Uneinigkeit (vgl. Kapitel 3), wobei unseres Erachtens von der Exklusivität einer Erklärungshypothese ohnehin nicht ausgegangen werden kann. Unter dem Stichwort der Zeitverdrängungshypothese (vgl. Kapitel 3.1) wurden Forschungsbefunde diskutiert, die davon ausgehen, dass es durch eine extensive Mediennutzung zum Einen zu einer zeitlichen Verdrängung von außerschulischen Lernaktivitäten und zum Anderen zu einem eingeengten Freizeitverhalten mit einer Einschränkung alltäglicher Lernprozesse und sozialer Kommunikationserfahrungen kommen kann.

Forschungsdaten weisen zudem darauf hin, dass sich insbesondere auch der Konsum gewalthaltiger Medieninhalte mittels seiner negativen Wirkung auf Aggressivität und aggressives Verhalten auch nachteilig auf Schulleistungen auswirken kann (Inhaltshypothese, vgl. Kapitel 3.2). Eine hohe Präferenz gewalthaltiger Medienangebote kann neben seiner Wirkung auf Aggressivität und aggressives Verhalten auch unmittelbar verantwortlich sein, dass schulische Lernprozesse beeinträchtigt werden (Löschungshypothese, vgl. Kapitel 3.3). Allen drei formulierten Hypothesen kommt dabei eine eigenständige Berechtigung bei der Erklärung einer verschlechterten Schulleistung durch spezifische Mediennutzungsmuster zu.

Die Schülerbefragung 2005 konnte die internationalen Befunde dahingehend recht eindrücklich bestätigen, dass sowohl die Ausstattung von Kindern mit Mediengeräten wie Fernseher und Spielkonsole als auch eine rein zeitlich überzogene Nutzung von Filmen und Computerspielen bereits deutlich die Gefahr schulischer Leistungseinbußen erhöht. Eine eigenständige Erklärungskraft kommt jedoch insbesondere der Nutzung gewalthaltiger Medienangebote zu. Vor allem Jungen, die bereits im Grundschulalter öfter als Mädchen mit Mediengeräten ausgestattet sind, höhere Nutzungszeiten als Mädchen aufweisen und gewaltbetonte Filme und Spiele stärker präferieren als Mädchen, scheinen hier besonders gefährdet zu sein. Sie verbringen häufig (zu) viel Zeit mit elektronischen Medien und konsumieren darüber häufig für sie ungeeignete Inhalte. 
Wenngleich sich universelle Wirkungsvermutungen dieser Art nicht ohne Einbezug weiterer Variablen aufrechterhalten lassen, ist die gegenteilige Annahme, dass nämlich Medien für sich genommen gar keinen näher bestimmbaren Einfluss haben sondern nur innerhalb eines individuellen Rezeptionsgeschehen eine subjektive Bedeutung gewinnen, ebenfalls als problematisch anzusehen. So konnte in der Schülerbefragung 2005 diesbezüglich gezeigt werden, dass die Folgen für das schulische Leistungsniveau sich durchaus auch dann zeigen, wenn relevante Einflussvariablen wie das Geschlecht und der Migrationsstatus des Kindes, das Bildungsniveau der Eltern sowie das innerfamiliäre Klima berücksichtigt werden.

Filmmedien und Computerspiele entfalten somit ein interindividuell stabiles, wenn auch differenziertes Wirkungsgeschehen auf ihre Nutzer. Besonders erwähnenswert ist dabei der Befund, dass Kinder mit protektiven Rahmenbedingungen, also Kinder ohne Migrationshintergrund aus Familien mit mittlerer und höherer Bildung, Kinder die sich von ihren Eltern geliebt fühlen und in den letzten vier Wochen keine Gewalterfahrungen gemacht haben, sogar besonders anfällig für die Folgen problematischer Mediennutzungsmuster zu sein scheinen. Eine qualitativ und quantitativ exzessive Mediennutzung wirkt sich in dieser Gruppe besonders nachhaltig auf die schulische Leistungsentwicklung aus, so dass diese nicht den Leistungslevel erreichen, der ihnen aufgrund ihres ansonsten positiven Umfeldes möglich wäre. Als bedeutsamer moderierender Einflussfaktor zeigte sich hier eine aktive Medienerziehung der Eltern. Diese hat eine mittelbare Auswirkung auf Schulleistungen, indem sie sowohl zu einer verringerten Präferenz für Mediengewaltinhalte als auch zu einer allgemeinen Reduktion der Mediennutzungszeiten führt, zwei wichtigen medialen Einflussfaktoren der Schulleistung.

Welche Maßnahmen lassen sich aus diesen Befunden ableiten? Ein erster wichtiger Ansatzpunkt ist die Vermittlung von Grundlagenwissen zu den Auswirkungen exzessiver und qualitativ problematischer Mediennutzung an Eltern und Pädagogen. Diese müssen stärker und einheitlicher als bisher über schädigende Medienwirkungen aufgeklärt werden. Ein großer Schritt wäre es bereits, wenn ein normativer Konsens erreicht würde, ein allgemein akzeptierter Maßstab sinnvollen Medienumgangs und notwendiger Medienerziehung verschiedener Alterstufen. Würde ein überwiegender Teil der Eltern und Lehrerschaft zu der Schlussfolgerung gelangen, dass bei einer frühen Ausstattung von Kindern mit allen erdenklichen elektronischen Medien nachteilige Wirkungen die Chancen bedeutend überwiegen, so wäre eine günstige Wirkung informeller Sozialkontrolle zu erwarten: Das, was für die meisten schon Gewissheit und gesellschaftlicher Konsens ist, wird letztlich auch für diejenigen Eltern zur verbindlichen Handlungsgrundlage und Norm, die individuell nicht davon zu überzeugen wären.

Um diesen Konsens zu gewährleisten sehen wir es für dringend notwendig an, die Vermittlung von Medienkompetenz weitaus stärker als bisher von - häufig gut gemeinten - Aktivitäten der Medienindustrie zu entkoppeln. Wirtschaftlich geförderte Medienkompetenzprogramme unterliegen fast immer - so gut sie auch konzeptionell gestaltet sind - dem Mangel, dass eine wichtige Form der Medienkompetenz gefährlich zu kurz kommt: Die Fähigkeit zum Verzicht und zur Selbstbeschränkung. 
Initiativen wie „Schulen ans Netz“ haben sich mit Sicherheit ihre Verdienste in der Ausstattung von Schulen mit moderner, multimedia- und internetfähiger Hardware erworben und auch viele sinnvolle Vorschläge in der Vermittlung technischer Kompetenzen an Lehrkräfte und Schüler/innen gemacht. Doch wer vermittelt Schülerinnen und Schülern, Lehrerinnen und Lehrern, wo die Grenzen und die Probleme der neuen Möglichkeiten liegen?

Projekte, die mithilfe einer Private-Public-Partnership zwischen staatlichen Bildungsinstitutionen und der Medienwirtschaft finanziert sind, werden immer technische Kompetenz, vielleicht auch inhaltliche Kompetenz von Schülerinnen und Schülern in den Vordergrund stellen, nie aber die Kompetenz zum Verzicht. Auch wenn es vielen in der Lehre Beschäftigten, besonders solchen, die sich in jahrelanger Arbeit mühevoll zusätzliche Kompetenzen im Umgang mit neuen Medien erarbeitet haben, nicht einleuchten mag: Keinesfalls ist die Vermittlung der Beherrschung von Medien wie z. B. Computern ein zentraler Baustein schulischer Bildung, um Kinder auf das Leben und den beruflichen Erfolg vorzubereiten und zentrale Kompetenzen zu entwickeln. Kinder müssen vielmehr kompetent darin werden, Medien kritisch, sinn- und maßvoll in ihr Leben zu integrieren. Ein erfolgreicher Informatiker wird man beispielsweise nicht, indem man bereits im Grundschulalter schneller zwischen den Tabs eines Interbrowsers hin- und her switchen kann, als die Augen der Lehrkraft erkennen können. Entscheidend ist hier vielmehr, dass man in der Lage ist, sich über lange Zeit hinweg strukturiert, konzentriert und lösungsorientiert mit einem komplexen Problem auseinanderzusetzen. Wie lange braucht es, einem Schüler der Mittelstufe die Bedienung eines aktuellen Messenger-Programms mit Videokonferenzfunktion beizubringen? Dreißig bis sechzig Minuten? Doch was nützt diese Kompetenz, wenn der Schüler zwei Jahre später im Bewerbungsgespräch scheitert, weil er die einfachsten Regeln der persönlichen Kommunikation nicht beherrscht?

Da Kinder nicht nur schon sehr früh über eine eigene umfassende Medienausstattung verfügen sondern sich darüber hinaus auch ganz von selber dafür begeistern, müssen Schulen diese Technologien weder zwingend bereitstellen noch einsetzen. Schulen müssen jedoch vermitteln und aufklären, in ähnlicher Weise, wie Schüler über andere Sachverhalte aufgeklärt werden wie z. B. im Sexualkundeunterricht über Sexualität oder über gesunde Ernährung im Biologieunterricht. Fragwürdige medienpädagogische Konzepte jedoch, die auf eine technische Beherrschung von Mediengeräten und Software ausgelegt sind, sind häufig nicht zielführend und medialisieren die Schule unnötig. Eine kompetenzsteigernde Wirkung ist durch die Ausstattung von Schulen oder Kindern mit Mediengeräten ohnehin nicht zu erwarten (vgl. PISA). Vielmehr ist zu befürchten, dass die Zeitverdrängungs-Hypothese auch in der Schule an Bedeutung gewinnt, indem die für wesentliche Lerngewinne notwendige Zeit durch den übermäßigen Medieneinsatz verschenkt wird.

Kinder sollten hingegen in der Schule das lernen, was ihnen der Alltag kaum mehr vermitteln kann: Auch ohne bunte Bilder und spannende Soundeffekte Informationen aufzunehmen, das Wesentliche in diesem Informationen zu erkennen, sie kritisch zu hinterfragen und sie schließlich eigenständig zu verarbeiten. Schulen sollten sich zu schade dafür sein (und sind es in der Tat auch), die Bedienung von 
Computersystemen und Programmen zu vermitteln, die in der späteren beruflichen Zukunft der Kinder keine Rolle mehr spielen werden, oder fragwürdige GoogleRecherchen im Internet zu unterstützen, die zumindest Kindern, die noch nicht über das notwendige Maß an Reflexionsvermögen verfügen können, eine oberflächliche Verarbeitung von Informationen regelrecht antrainieren. Vielmehr sollten sich Schulen wieder verstärkt um die Förderung leistungsbezogener und sozialer Basiskompetenzen bemühen.

Das bedeutet nicht, dass Medienunterricht keine Relevanz im schulischen Alltag hat. Er muss nur deutlich andere Schwerpunkte setzen. Medientagebücher können jungen Schülerinnen und Schülern helfen, die Bedeutung der Medien in ihrem Alltag zu erkennen. Die Analyse der Handlungs- und Personenstereotypen in FirstPerson-Shootern kann älteren Schülern helfen, kritisch über diese Art der Computerspiele nachzudenken. Fernsehfreie Tage machen den Blick frei für alternative Möglichkeiten der Freizeitgestaltung. Dabei handelt es sich keineswegs um die bewahrpädagogischen Träumereien aus dem Elfenbeinturm. Es geht dabei auch keinesfalls um die Verbannung neuer Medien aus dem Leben von Schülerinnen und Schülern, sondern darum, Prioritäten zu setzen.

Die Medienwirkungsforschung zu Mediengewalt und Aggressivität nimmt im theoretischen Teil dieses Buches und unseren eigenen Analysen zu Recht einen hohen Stellenwert ein (vgl. Kapitel 3.2). Die Forschung weist darauf hin, dass insbesondere die von männlichen Kindern und Jugendlichen bevorzugten Angebote bei Filmen und Computerspielen einen hohen Gewaltanteil aufweisen. Insgesamt ist zudem der Gewaltanteil im Fernsehen ansteigend und auch der Gewaltrealismus in Computerspielen nimmt Jahr für Jahr zu. Bezüglich der Wirkungen dieser Inhalte wird die Forschungslage zunehmend differenzierter. Für Aggressivität und Gewalthandeln relevante Faktoren wie z. B. aggressionsbezogene Verhaltensskripte oder die Fähigkeit zum Mitleid mit Opfern werden gerade durch einen längerfristigen und regelmäßigen Gebrauch von medialen Gewaltangeboten negativ beeinflusst. Wenngleich immer auch andere Variablen einen Einfluss auf diesen Zusammenhang nehmen, kann an der grundsätzlich aggressionssteigernden Wirkung gewalthaltiger Medienangebote nicht mehr gezweifelt werden. In der Literatur wird zudem begründet dargelegt, dass beim interaktiven Medium Computerspiel ein besonders hohes Wirkpotential zu befürchten ist.

Unsere Daten bestätigen die internationalen Befunde und erweitern sie um einen zentralen Aspekt: Sie zeigen auf, dass gewalthaltige Medien nicht nur für aggressionsnahe Konstrukte eine Rolle spielen, sondern auch für tatsächliches Gewaltverhalten im Alltag von zentraler Bedeutung sind. Innerhalb des Beziehungsgeflechtes, welches als maßgeblich für die Gewaltprävalenz gelten kann tritt das Spielen von Computerspielen, die erst ab 16 oder ab 18 Jahren frei gegeben sind, als eigenständiger Faktor mit unmittelbarem Einfluss auf die Gewaltprävalenz in Erscheinung (vgl. Kapitel 4.4.2, Abbildung 44). In zusätzlichen bivariaten Analysen zeigt sich zwar, dass auch die Rezeption gewalthaltiger Filme deutlich mit erhöhter Gewaltprävalenz korreliert. Diese verliert aber im Wechselspiel mit anderen maßgebli- 
chen Faktoren für die betrachteten Jugendlichen im Alter von durchschnittlich 15 Jahren an Bedeutung und scheint mehr ein Indikator für erhöhte Gewaltakzeptanz und einen mit Gewaltakzeptanz verknüpften Lebensstil als unmittelbarer kausaler Faktor zu sein.

Insgesamt zeigt sich, dass die Computerspiele trotz ihres eigenständigen Erklärungswertes für Gewaltprävalenz in ihrer Relevanz erwartungsgemäß hinter anderen Faktoren zurücktreten. Von übergeordneter Bedeutung sind die normative Überzeugungen des Jugendlichen, inwieweit Gewalt als legitimes Mittel zur eigenen Zielerreichung erachtet wird (Gewaltakzeptanz) und das Vorhandensein eines bereits delinquenten Freundeskreises. Gewaltakzeptanz steht wiederum in Wechselwirkung mit dem Spielen von gewalthaltigen Spielen, indem sowohl eine erhöhte Gewaltakzeptanz die Zuwendung zu den Spielen erhöht als auch das Spielen der Spiele eine höhere Akzeptanz von Gewalt fördert. Insgesamt bestätigen unsere Erkenntnisse die Annahme, dass die Bevorzugung von Gewaltmedien - und bei unserer Population insbesondere das Spielen gewalthaltiger Computerspiele - Dispositionen zu Aggressivität und Delinquenz festigen können und damit im Rahmen eines allgemeinen delinquenten Lebensstiles stehen (vgl. Lösel \& Bliesener, 2003, S. 176).

Welche Folgerungen ergeben sich aus diesen Befunden? Zunächst unterstreichen die Daten die Notwendigkeit eines effektiven Jugendmedienschutzes. Die Nutzung nicht altersgerechter Spiele durch Kinder und Jugendliche muss besonders kritisch gesehen werden. Dies wird umso deutlicher, wenn man sich vergegenwärtigt, dass bei Neuntklässlern mit einem Durchschnittsalter von 15 Jahren das Spielen von Spielen ab 16 Jahren bereits einen nicht zu vernachlässigenden Erklärungswert für Gewaltprävalenz besitzt. Dieser Befund lässt die Validität der Alterseinstufung „Ab 16 Jahren“ zudem dahingehend als fragwürdig erscheinen, dass die Daten ein entwicklungsschädigendes Potential dieser Spiele für eine Population unterstreichen, die im Durchschnitt das geforderte Mindestalter nur unmaßgeblich unterschreitet. Bedenkt man zudem, dass diese Spiele bereits von einem guten Fünftel aller Grundschüler vierter Klassen regelmäßig genutzt werden, ergeben sich weitere Zweifel daran, ob die Alterseinstufungspraxis bei Spielen mit einer Freigabe ab 16 Jahren den Erfordernissen des Jugendschutzes angemessen Rechnung trägt.

Eine weitere zentrale Forderung ergibt sich aus einem Befund, der nur auf den zweiten Blick aus unseren Daten erkennbar wird: Es zeigt sich, dass eine erhöhte Risikosuche beim Jugendlichen sowohl die Gewaltakzeptanz erhöht als auch die Nutzung von gewalthaltigen Computerspielen. Risikosuche ist zunächst lediglich ein Anzeichen dafür, dass der Jugendliche gerne seine Grenzen austesten will, gerne Risiken eingeht oder bereit ist, sich für das Erleben von Abenteuern in Gefahr zu begeben, für sich also ein neutrales Persönlichkeitsmerkmal dem Sensation Seeking nahe stehend. Dass eine erhöhte Risikosuche sowohl die Gewaltakzeptanz erhöht als auch das Spielen gewalthaltiger Spiele fördert könnte ein Hinweis dafür sein, dass Jugendlichen in unserer Gesellschaft geeignete Angebote fehlen, ihren Drang nach Abenteuerlust auszuleben. Die Flucht in Gewaltspiele könnte damit Ausdruck eines Mangels sein, dem mit angemessenen Freizeitangeboten begegnet werden könnte. 
Wie dargestellt wurde ist sich die Forschung noch uneinig darüber, ob exzessivem Computerspielen eine eigenständige klinische Bedeutsamkeit zukommt und wenn ja, ob diese zutreffend als Verhaltenssucht einzustufen ist oder alternativ anderen Störungsbildern untergeordnet werden kann (vgl. Kapitel 3.2). Daher fällt auch die Definition von Computerspielsucht in der Literatur bislang sehr uneinheitlich aus und Prävalenzschätzungen bewegen sich je nach Studie, Untersuchungsmethode und Stichprobe zwischen 3 und 9 Prozent. Unsere Daten bestätigen jedoch insgesamt die hohe Relevanz und Notwendigkeit dieser Forschungsbemühungen. So hat sich für unsere Stichprobe von befragten Neuntklässlern gezeigt, dass diese mit zunehmender Spielzeit in erhöhtem Maße auch Kennzeichen für eine Computerspielsucht erfüllt. Viel- und Exzessivspieler erscheinen also verstärkt als süchtig, und berichten Entzugserscheinungen bzw. eine starke gedankliche Fokussierung auf das Spielen, einen Kontrollverlust hinsichtlich der Spielzeit und ein fortgesetztes Spielen trotz negativer Konsequenzen für Schule und Privatleben.

Wenngleich diese drei Indikatoren noch keinen allumfassenden bzw. erschöpfenden Einblick in das Phänomen Computerspielsucht erlauben, sprechen sie doch recht deutlich für eine Pathologie und klinische Relevanz der zugrunde liegenden Mechanismen. Insgesamt findet sich unter den Jugendlichen ein Anteil von 1,5 Prozent der als süchtig und 3 Prozent der als gefährdet einzustufen ist. Damit bewegt sich unsere Prävalenzschätzung im Vergleich zu anderen Studien im unteren Bereich. Zu bedenken ist hierbei, dass unsere Daten auf einem für vielfältige Befragungsregionen repräsentativen Querschnitt von Jugendlichen 9. Schulklassen beruhen, unter den Jungen wie Mädchen und Viel- wie Nichtspieler unterschiedlicher Schulstufen fallen. Dadurch weist unsere Stichprobe für Prävalenzschätzungen eine weitaus höhere Güte auf als andere Befragungen, die zum Teil vorab nur einen Ausschnitt bestimmter Spielerpopulationen betrachten oder die Anwerbung bereits über einen bestimmten nicht unmaßgeblichen Kontext vornehmen (z. B. das Internet). Gleichzeitig ist festzustellen, dass unsere Neuntklässlerstichprobe mit einem Durchschnittsalter von 15 Jahren eine Altersklasse repräsentiert, die besonders hohe Computerspielnutzungszeiten aufweist. Es ist daher davon auszugehen, dass unsere Prävalenzschätzungen das Ausmaß von Computerspielsucht unter Kindern und Jugendlichen vermutlich eher über- als unterschätzen.

Die insgesamt geringere Prävalenz für Computerspielsucht kann aber nicht über spezifische Probleme hinwegtäuschen, auf die unsere Daten ebenfalls hinweisen: Gerade die Jungen erscheinen wieder in besonderer Weise als Sorgenkinder, indem sie gegenüber Mädchen einen weitaus höheren Anteil von Vielspielern und Exzessivspielern aufweisen. Vielspieler und Exzessivspieler wiederum werden gegenüber Normalspielern durch eine besonders hohe Suchtgefährdung auffällig. Verschärft wird die Kluft zwischen Mädchen und Jungen nun zusätzlich dadurch, dass vielspielende Jungen, die im Durchschnitt genauso lange spielen wie eine Vergleichsgruppe vielspielender Mädchen einen fast viermal höheren Anteil an Süchtigen und einen fast fünfmal höheren Anteil an Suchtgefährdeten aufweist. Auch bei den Exzessivspielern ist der Anteil von Süchtigen fast viermal so hoch wie bei Exzessivspielerinnen, der Anteil von Gefährdeten immerhin dreimal so hoch. Dieser Befund ist neu 
und in dieser Form in der Literatur noch nicht berichtet worden. Damit wird das besondere Ausmaß deutlich, in dem Jungen von Computerspielen abhängig werden können.

Von maßgeblichem Einfluss erscheint auch das gewählte Spiel bzw. Spielgenre. In einem prototypischen Vergleich dreier Computerspiele konnten wir zeigen, dass bestimmte Spiele höhere Nutzungszeiten nahe legen und Spieler bestimmter Spiele damit häufiger den Viel- und Exzessivspielern zugerechnet werden müssen. Ein besonders überraschendes Ergebnis: Spieler bestimmter Spiele weisen auch unabhängig von ihrer Nutzungszeit ein höheres Risiko für Computerspielabhängigkeit auf, da offenbar die Spiele selbst ein stärkeres Suchtpotential aufweisen. Spiele, die vornehmlich online mit oder gegen andere menschliche Spieler gespielt werden (Beispiel Counter Strike) und darunter insbesondere Spiele des MMORPG-Genre, welche die Einnahme komplexer Rollen innerhalb hochgradig authentischer Welten ermöglichen (Beispiel World Of Warcraft), scheinen ein besonders hohes Abhängigkeitspotential auf ihre Nutzer zu entfalten. Die Vermutung, dass gerade bestimmte Spiele in besonders hohem Maße abhängig machen können wurde vielfach geäußert und wird immer wieder intensiv in Spielercommunities diskutiert (Beispiel World Of Warcraft). Unsere Daten liefern nun einen ersten empirischen Nachweis dafür, dass offenbar tatsächlich das Spielen bestimmter Spiele mit höheren Nutzungszeiten und auch unabhängig davon mit höheren Suchtwerten einhergeht. Offen bleibt jedoch, inwieweit ausschließlich die Spiele diese Wirkung entfalten, oder ob sich nicht bestimmte Nutzer, die bereits eine besondere Prädisposition für exzessives bzw. süchtiges Spielverhalten aufweisen, auch gezielt bestimmten Spielen zuwenden.

Welche Implikationen ergeben sich aus diesen Befunden? Zunächst einmal erscheint es notwendig, die klinische Bedeutsamkeit pathologischer Computerspielnutzung nachhaltig aufzuklären und in der Nosologie zu etablieren. Dafür ist es wichtig, den Begriff der Computerspielsucht einheitlich zu definieren und von anderen Störungsbildern abzugrenzen und in Folge dessen Screeninginstrumente zu entwickeln, mit denen es Klinikern möglich wird, das Vorliegen einer Computerspielsucht im Einzelfall zuverlässig abzuklären. Frühwarnkriterien müssen Eltern und Pädagogen in die Lage versetzen, ein lediglich leidenschaftliches zeitlich nicht überdauerndes Spielen von einer beginnenden Computerspielsucht abzugrenzen. Leitfäden und Handbücher könnten Eltern und Pädagogen geeignete Maßnahmen im Umgang mit computerspielsüchtigen Kindern und Jugendlichen an die Hand geben. Hier ist ein erschreckender Mangel festzustellen. Zusätzlich kann darüber nachgedacht werden, Spiele, die hohe Nutzungszeiten und damit ein erhöhtes Suchtpotential erwarten lassen auch gesondert auf der Produktverpackung zu kennzeichnen. Darunter fällt sicherlich ein hoher Anteil aller Spiele, der sich vernetzt mit anderen online spielen lässt und insbesondere das Genre der MMORPG. Insgesamt ist jedoch über zentrale pädagogische Maßnahmen nachzudenken, die auch präventiv in schulischen Vermittlungsformen innerhalb medienpädagogischer Unterrichtskonzepte zum Einsatz kommen können. Kindern muss schon früh nahe gebracht werden, in welcher Weise Medien und insbesondere Computerspiele verführerische Gratifikationserlebnisse vermitteln können, die vom Nutzer auch missbräuchlich genutzt 
werden können. Kinder sollten früh dafür sensibilisiert werden, wie viel Zeit sie in virtuellen und nicht virtuellen Erlebniswelten verbringen und welche Folgen das für sie und ihre eigenen Entwicklung haben kann. 